\title{
Pedundulatory Robotic Locomotion: Centipede and Polychaete Modes in Unstructured Substrates
}

\author{
Michael Sfakiotakis and Dimitris P. Tsakiris
}

\begin{abstract}
The present paper considers a novel class of robotic systems, termed pedundulatory locomotors, which can be thought of as undulatory robots augmented by multiple pairs of lateral paddle-like appendages ("parapodia"). Bioinspired strategies for synchronizing the movement of the parapodia with the body undulations, emulating organisms like the centipedes and the polychaete worms, are presented, giving rise to distinct pedundulatory modes. These modes are investigated and comparatively assessed, both in simulation and via experiments with the Nereisbot prototype locomoting on sand and on several other unstructured substrates. Our studies demonstrate the rich gait repertoire and enhanced performance of pedundulatory systems, compared to purely undulatory ones.

Keywords - biomimetic robotics, undulatory locomotion, motion control, polychaete annelids, centipedes.
\end{abstract}

\section{INTRODUCTION}

The present paper considers the development of two distinct bio-inspired motion control strategies, for a novel class of robotic systems, which are based on the synergetic action between body undulations and movements of several lateral active paddle-like appendages (called parapodia). This type of locomotion has been named pedundulatory [1] (the term is derived by prefixing the word undulatory with the Latin root ped-, which means "pertinent to feet") and can furnish the robotic system with a rich gait repertoire, as well as enhanced locomotion performance, particularly with regard to movement over a variety of challenging environments. Therefore, it merits special attention, since it could benefit emerging robotic applications ranging from novel diagnostic systems for healthcare (e.g., endoscopic access to the human gastrointestinal tract) to robotic tools for search-and-rescue operations and to planetary exploration.

The two proposed pedundulatory modes of robotic locomotion are inspired by the locomotion of the polychaete annelid marine worms (bristleworms) and of the chilopoda terrestrial arthropods (centipedes). Despite sharing a fairly similar body plan, characterized by a large number of lateral appendages distributed across their elongated, segmented and flexible bodies, polychaete and centipedes appear to utilize these features in a different manner for terrain traversal or terrain manipulation. This brings up the issue of replicating their locomotion strategies by robotic analogues, and exploring the suitability of each strategy for various substrates.

This work was supported in part by the European Commission, through the IST project VECTOR (FP6-033970).

The authors are with the Institute of Computer Science, Foundation for Research and Technology - Hellas, N. Plastira 100, Vassilika Vouton, GR70013, Heraklion, Greece. The first author is also with the Dept. of Electrical Engineering, Technological Education Institute of Crete, Heraklion, Greece. \{sfakios, tsakiris\}eics.forth.gr.

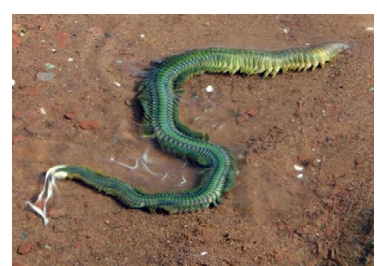

(a)

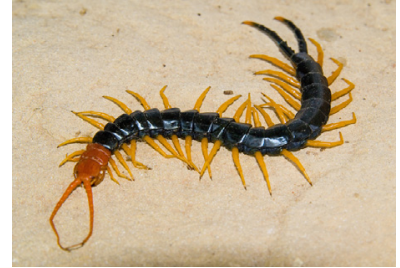

(b)

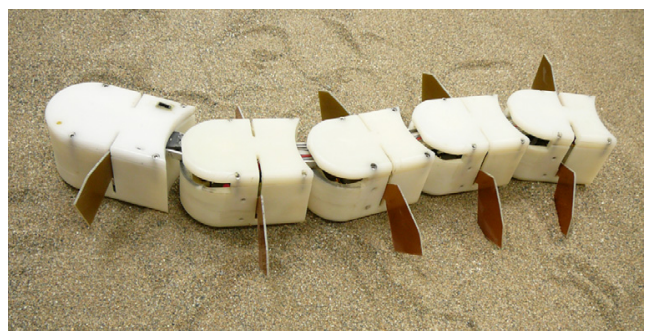

(c)

Fig. 1. (a) Nereis virens, a polychaete annelid marine worm. (b) Scolopendra heros, a centipede. (c) The Nereisbot pedundulatory robotic prototype.

Investigations in this direction are carried out both via simulations and via experimental studies with the Nereisbot pedundulatory robotic prototype (Fig. 1c). The present paper advances related work in [1] introducing new (centipede-like) motion control strategies, utilizing an ameliorated robotic prototype (more sets and enhanced design of parapodia, redesigned electronics), improved computational models, and more thorough parametric studies, including locomotion experiments on a variety of unstructured substrates (sand, grass and ploughed soil).

The development of these pedundulatory systems builds upon our previous work [2]-[5], which has identified, and started replicating in the context of propulsion over granular substrates, the intriguing locomotion of the polychaete annelids. These works consider purely undulatory robots, i.e., ones without parapodia, mostly investigating polychaetelike tail-to-head body undulations. Relevant work includes other research efforts on non-wheeled undulatory locomotion (e.g., [6]-[12]), as well as the salamander-like robot [13], which employs for land locomotion a standing body wave, combined with the coordinated movement of its four limbs.

Section II of the paper outlines the biological inspiration for the developed motion control schemes. Section III presents the body mechanics of pedundulatory systems and their interaction with the locomotion environment, while the polychaete-like and centipede-like pedundulatory modes are developed and demonstrated via simulations in Section IV. The Nereisbot prototype is described in Section V. 
Experimental results, presented in Section VI, demonstrate the efficacy of pedundulatory locomotion over different substrates, and verify the validity of our computational models.

\section{BIOLOGICAL INSPIRATION}

The polychaete annelids are marine worms, which can be found living in the depths of the ocean, floating near the surface, or burrowing in the mud and sand of the seashore (Fig. 1a). Their hydrostatic skeleton comprises a large number of segments, each one featuring a pair of laterally-extending paddle-shaped active appendages, called parapodia. When crawling rapidly, polychaete combine direct (i.e., tail-to-head) body undulations (opposite direction of propagation than in snakes or eels) with elliptical motions of the parapodia, occurring in two-stroke cycles: during the power stroke, the parapodia move backwards, pushing against the substrate to generate propulsive forces; then, during the recovery stroke, they are lifted off the ground and brought forward, for the next cycle to commence. This parapodial activity occurs in waves propagating from the posterior to the anterior, along the two sides of the worm, with the two parapodia of each segment moving in antiphase. It is timed so that the power stroke (resp. recovery stroke) of each parapodium occurs at the crest (resp. trough) of the tail-to-head body wave. This locomotion strategy is also employed by polychaete for swimming [14]-[16].

The centipedes are terrestrial arthropods, characterized by one pair of long, articulated legs per leg-bearing segment (Fig. 1b). The legs are capable of up-down flexion and forward-backward bending, and their movements are, as in polychaete, combined with body undulations for terrestrial locomotion. However, the locomotion strategy in centipedes is reversed, as they employ retrograde (i.e., head-to-tail) body waves (like snakes and eels), while their leg movements are timed so that ground contact occurs at the trough of the body wave [17]-[19]. The few centipede species capable of swimming, do so purely by head-to-tail undulations, with the legs held against the sides of the body [19].

\section{COMPUTATIONAL MODELING}

\section{A. Mechanical Model of Pedundulatory Systems}

A mechanical model of the Nereisbot pedundulatory prototype, with 14 actuated degrees of freedom, is shown in Fig. 2. It is composed of five links (link-1 is the tail, and link- 5 is the mechanism's head), interconnected by a total of four planar rotary joints $J_{i}$, whose actively-controlled joint angles are denoted by $\phi_{i}$, while the distance between two consecutive joints is denoted by $l$. On each segment, a pair of laterally placed parapodial links of length $l_{p}$ is mounted at a distance $b$ from the segment's left-most edge. The parapodia are connected to the segment via planar dorsoventrally oriented single-degree-of-freedom rotary joints, whose angles to the horizontal are denoted by $\zeta_{i}$ and $\chi_{i}$, for the left-hand $\left(P_{L, i}\right)$ and right-hand $\left(P_{R, i}\right)$ parapodium of the $i$ th segment, respectively. The overall desired "paddling" action of the parapodia then emerges when these up-down movements are combined with body undulations. This configuration leads to

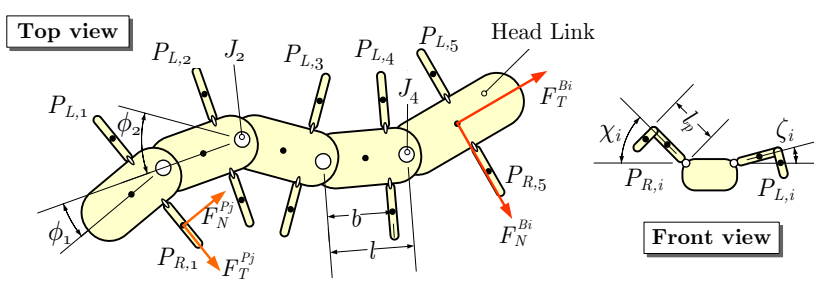

Fig. 2. Model of the Nereisbot robotic prototype.

a significant simplification of the mechanical design; however, it also entails that, unlike its biological counterparts, the mechanism at hand cannot propel itself by parapodial actions alone. Finally, by retracting all parapodia, the mechanism is essentially reduced to an undulatory robot.

A computational model of the above system, incorporating the mechanical parameters (masses, dimensions, etc.) of the actual Nereisbot prototype, has been implemented in the SIMUUN simulation environment [20], which is based on the SimMechanics toolbox of Matlab/Simulink.

\section{B. Interaction with the Environment}

Since the pedundulatory mechanism is assumed to crawl over the locomotion substrate (rather than being supported by the parapodial appendages), our computational models need to account for the interaction with the environment of both the body segments and the parapodia.

Body undulations: Apart from providing the means to position the parapodia and move them with respect to the substrate, body undulations also play an active role in motion generation, much like in any undulatory system. Indeed, by retracting the parapodia, the pedundulatory mechanism can also locomote by body undulations alone, through the coupling of the mechanism's internal shape changes to external motion constraints (the frictional forces applied through the interaction with the locomotion environment). The interaction of the undulatory mechanism's $i$ th body segment $\left(B_{i}\right)$ with the locomotion environment is described here using a Coulomb-like force model, involving the decoupled components $F_{T}^{B i}=-\mu_{T} m g \operatorname{sgn}\left(v_{T}^{B i}\right)$ and $F_{N}^{B i}=$ $-\mu_{N} m g \operatorname{sgn}\left(v_{N}^{B i}\right)$, acting in the tangential and normal direction of segment motion, where $v_{T}^{B i}$ and $v_{N}^{B i}$ are the respective velocity components, while $m$ is the segment's mass and $g$ is the constant of gravity. The tangential $\left(\mu_{T}\right)$ and normal $\left(\mu_{N}\right)$ Coulomb friction coefficients depend on the shaping and material of the links' underside, as well as on the material properties of the substrate. In general, the larger the differential between these coefficients, the larger the stride length (distance traveled per undulation cycle) attainable for a given body wave. When $\mu_{N} / \mu_{T} \gg 1$, the undulatory system moves in the direction opposite to that of the wave propagation, so that forward propulsion is by retrograde (head-to-tail) body waves (see, e.g., [4], [9]-[12], [21]-[23]). By contrast, when $\mu_{N} / \mu_{T}<1$, or when $\mu_{N} / \mu_{T} \simeq 1$ (as is the case in our system), the overall locomotion is along the body wave direction, so that forward motion is by direct (tail-to-head) waves (for analysis and relevant robotic implementations, see [1]-[3], [6], [8]). 
Parapodia: As a first approximation, the simple Coulomblike frictional force model $F_{T}^{P j}=-f_{T}^{i} \operatorname{sgn}\left(v_{T}^{P j}\right), F_{N}^{P j}=$ $-f_{N}^{i} \operatorname{sgn}\left(v_{N}^{P j}\right)$ (see Fig. 2), may be used to describe the interaction of the $j$ th parapodium $\left(P_{j}\right)$ with sand [1]. The magnitudes of the frictional forces in the normal $\left(f_{N}^{i}\right)$ and tangential $\left(f_{T}^{i}\right)$ direction can be determined experimentally for each pair of parapodia placed on the robot's $i$ th segment.

\section{MOTION CONTROL}

\section{A. Pedundulatory Gait Generation}

In the mechanical system of Fig. 2, parapodial movements are limited to single-plane dorsoventral motions, and, hence, cannot generate locomotion in the absence of body undulations. Propulsive forces may then be imparted by appropriately timing the parapodial activation with respect to the propagation of the body wave, so that ground contact occurs when the parapodia are moving backwards with respect to the substrate.

Polychaete mode: A tail-to-head body wave may be propagated across the mechanism by prescribing the temporal variation of the $i$ th body joint angle as:

$$
\phi_{i}(t)=A \sin \left(2 \pi f t+(N-i) \phi_{\text {lag }}\right)+\psi, i=1, . ., N-1,
$$

where the $N-1$ body joint angles vary sinusoidally, with a common amplitude $A$, frequency $f$, angular offset $\psi$ and a constant phase lag $\phi_{\text {lag }}>0$ between consecutive joints. For $\phi_{\text {lag }}=2 \pi / N$, there is one complete wavelength of the undulatory wave propagating across the mechanism. When $\psi=0$, propulsion is along a straight line, while curved paths are obtained for $\psi \neq 0$. Note, that, for purely undulatory gaits, in order for the above body joint controls to generate an overall forward motion (i.e., with the mechanism moving "head first"), the interaction of the body segments with the locomotion environment must be such that resistance to lateral segment motions is (considerably) less than resistance to tangential motions.

As described in Section II, in the biological analogue of the polychaete, such tail-to-head body undulations are synchronized with the parapodial activity, so that the thrustproducing power stroke (resp. recovery stroke) of each parapodium occurs at the crest (resp. trough) of the body wave. For the mechanical system at hand (Fig. 2), this may be achieved by the following alternating activation of the right and left parapodium of the $j$ th body segment (note that the parapodia are in contact with the substrate for $\chi_{j}(t)=0$ and $\zeta_{j}(t)=0$, respectively):

$$
\chi_{j}(t)=\left\{\begin{array}{ll}
0, & \text { for } \beta_{j}(t)>r_{j} \\
A_{p}, & \text { otherwise }
\end{array}, \zeta_{j}(t)=\left\{\begin{array}{ll}
0, & \text { for } \beta_{j}(t)<-r_{j} \\
A_{p}, & \text { otherwise }
\end{array},\right.\right.
$$

$$
\text { for } \beta_{j}(t)=\sin \left(2 \pi f t+\left(N+g_{j}^{p}-j\right) \phi_{\text {lag }}\right), j=1, . ., N \text {, }
$$

where $r_{j}$ is an appropriate threshold $\left(0<r_{j}<1\right)$ and $A_{p}$ determines the angle at which the parapodia are retracted, when not in contact with the substrate. The periodic movements of each segment's pair of parapodia are synchronized with the body wave via the sinusoidal reference signal $\beta_{j}(t)$. The latter is derived by the (scaled) corresponding body joint angle $\phi_{i}(t)$, appropriately shifted in time via the constant $g_{j}^{p}$ (which depends on the relative placement of the parapodia on the segment with respect to the joint, see Fig. 2), to ensure its temporal variation corresponds to that of the parapodium velocity, resolved along the mean direction of propulsion.

Centipede mode: In centipede locomotion, the legs make contact with the substrate when the corresponding body segment is in the trough of the head-to-tail body wave. The latter may be obtained by:

$$
\phi_{i}(t)=A \sin \left(2 \pi f t+i \phi_{\text {lag }}\right)+\psi, i=1, . ., N-1 .
$$

Note that, for purely undulatory gaits, if resistance to lateral segment motions is (considerably) less than resistance to tangential motions, (3) will generate an overall backwards motion (i.e., with the mechanism moving "tail first").

In centipede mode, the movements of the parapodia are synchronized with the head-to-tail body wave as follows:

$\chi_{j}(t)=\left\{\begin{array}{l}0, \text { for } \beta_{j}(t)<-r_{j} \\ A_{p}, \text { otherwise }\end{array}, \zeta_{j}(t)=\left\{\begin{array}{l}0, \text { for } \beta_{j}(t)>r_{j}, \\ A_{p}, \text { otherwise }\end{array}\right.\right.$

for $\beta_{j}(t)=\sin \left(2 \pi f t+\left(j-g_{j}^{c}\right) \phi_{l a g}\right), j=1, . ., N$.

Turning gaits: For both pedundulatory modes, turning motions may be instigated by a number of different ways, e.g., (a) by introducing an angular offset $\psi \neq 0$ in the body wave (1) or (3), while the parapodia operate in the normal bilateral fashion of the corresponding pedundulatory mode, (b) by unilateral parapodial activations, while $\psi=0$, and (c) by combining a value of $\psi \neq 0$ with unilateral parapodial activation. The "unilateral" parapodial activation in these methods implies retracting all parapodia in the side of the mechanism appropriate for the desired turning direction.

\section{B. Simulation Studies}

This Section presents a series of simulations of the SIMUUN Nereisbot model, implementing the polychaete (1)(2) and centipede (3)-(4) pedundulatory modes.

Initially, simulations of these two modes with the robot "lifted in the air", i.e., in the absence of any external frictional forces (leading to in-place pedundulations of the system, without any net movement), were carried out, allowing insights regarding the movement and coordination of the parapodia. The obtained data may be subsequently analyzed to fine-tune, for each pair of parapodia, the parameters $g_{j}^{p}$ and $g_{j}^{c}$ in (2) and (4), respectively. Figures 3 and 4 illustrate the kinematics of the two proposed pedundulatory modes (when $\phi_{\text {lag }}=2 \pi / N$ ), indicating that, for both motion control schemes, the parapodia move backwards relative to the substrate during their power stroke (and, hence, may generate positive thrust during ground contact).

Subsequently, simulations were performed of Nereisbot implementing the two proposed pedundulatory modes, as well as the purely undulatory mode (i.e., with retracted parapodia), for locomotion over sand. Data from appropriate 


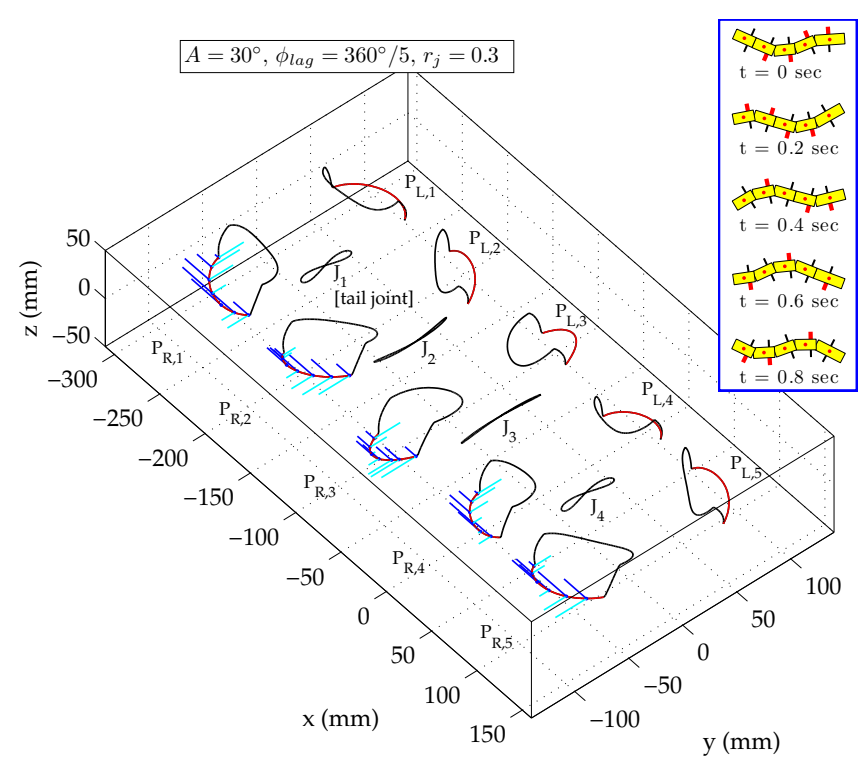

Fig. 3. Polychaete-like pedundulatory mode: Main figure - Trajectories traced by the parapodia tips, during a cycle of in-place polychaete-mode pedundulations. The part of the traces shown in red indicates the power stroke of the parapodia, when they are in contact with the substrate. The blue and cyan lines denote the $x-$ and $y$ - componenets of the parapodium velocity during the power stroke, indicating that the velocity is mostly directed towards the tail. The trajectories of the four body joints $J_{1}-J_{4}$ are also shown on the plot. Upper right inset - Snapshots of the mechanism during the pedundulation cycle. The parapodia which are in contact with the ground are denoted by thick red lines. The plot illustrates how both the body wave and the parapodial activity propagates from the tail (leftmost segment) to the head (rightmost segment) of the robot.

frictional force measurements (see Section VI) were employed to specify the parameters of the force models (as per Section III-B) for the interaction with the environment of the body segments $\left(\mu_{T}=0.518\right.$ and $\left.\mu_{N}=0.463\right)$ and of the parapodia $\left(f_{N}^{i}=1.8 \mathrm{~N}\right.$ and $\left.f_{T}^{i}=0.06 \mathrm{~N}\right)$. Note that, for these values of $\mu_{T}$ and $\mu_{N}$, forward locomotion in the purely undulatory mode is by tail-to-head body waves. Indicative trajectories for the forward and turning gaits are shown in Fig. 5. Furthermore, the results of a parametric study with this simulation setup, over a range of values for the joint oscillation amplitude $A$, are provided in Figs. 9c and $11 \mathrm{c}$, in the context of comparing them with the experimental data.

\section{THE NEREISBOT PROTOTYPE}

The proposed pedundulatory modes are investigated experimentally with an updated variant of the Nereisbot robotic prototype [1]. Nereisbot comprises a total of five body segments (fabricated from plastic), each equipped with a pair of parapodial appendages, providing a total of 14 degrees-offreedom (corresponding to 4 body joints and 10 parapodial joints). The first four body segments are identical (length $100 \mathrm{~mm}$, width $50 \mathrm{~mm}$, mass $270 \mathrm{~g}$ ), and each one encases three motors, actuating three rotary joints: a high-torque R/C servo unit (HiTech HSR-5995TG) drives the main body joint, through which consecutive segments are connected together, while a pair of smaller R/C servos (HiTech HS$81 \mathrm{MG}$ ), placed on either side of the main body joint, are used to independently drive the segment's parapodia. The fifth

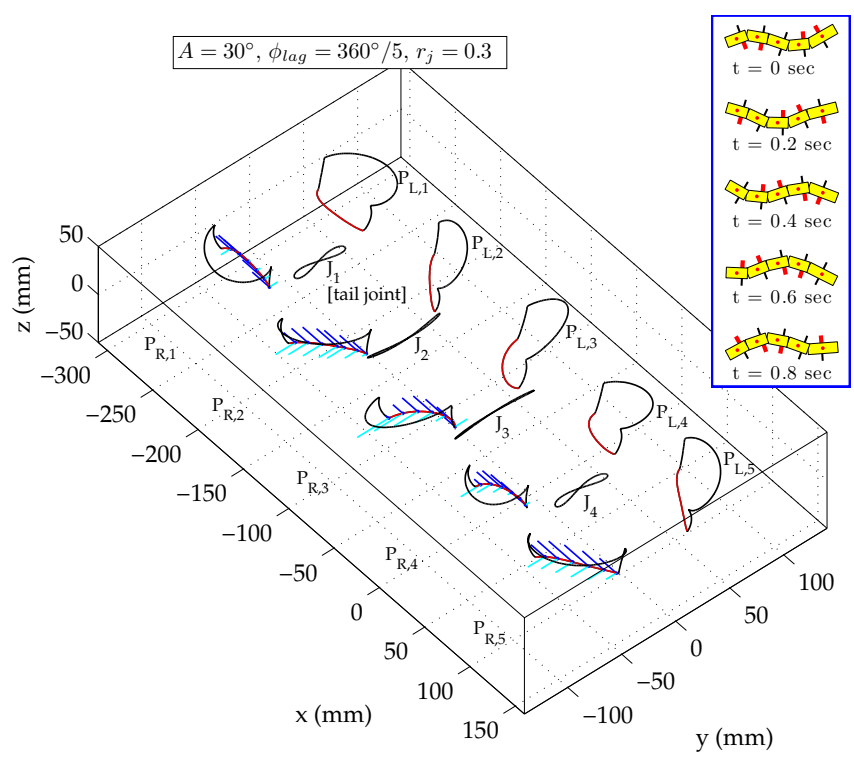

Fig. 4. Centipede-like pedundulatory mode: Main figure - Trajectories traced by the parapodia tips, during a cycle of in-place centipede-mode pedundulations. Upper right inset - Snapshots of the mechanism during the pedundulation cycle, illustrating how both the body wave and the parapodial activity propagates from the head to the tail of the robot. (see caption of Fig. 3 for a detailed explanation of the figure's elements)

segment of the robot, designated as the "head" segment, does not require a body joint servo, and incorporates just the pair of parapodia-driving motors. It has been made longer (length $140 \mathrm{~mm}$, width $50 \mathrm{~mm}$, mass $280 \mathrm{~g}$ ), in order to accommodate the controller board, which employs a Motorola DSP56807 microcontroller for generating all the control signals to the motors implementing the pedundulatory movements.

The parapodia, which are directly mounted on the shaft of their driving motor so that their flat surface is lateral to the segment's main axis, are lightweight and capable of

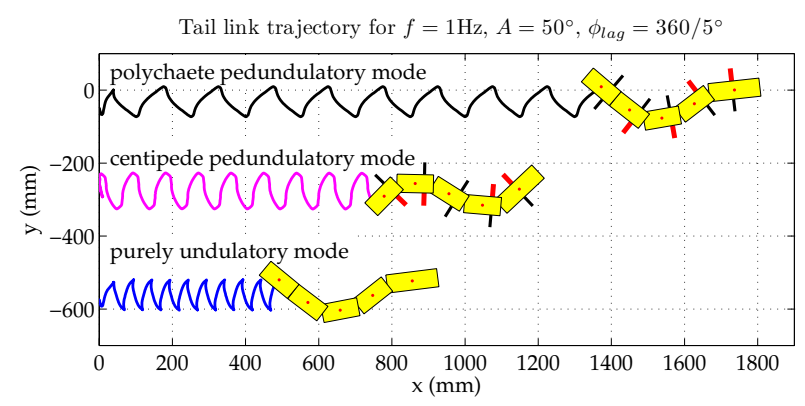

(a) forward gait simulations (10 cycles shown for each mode)

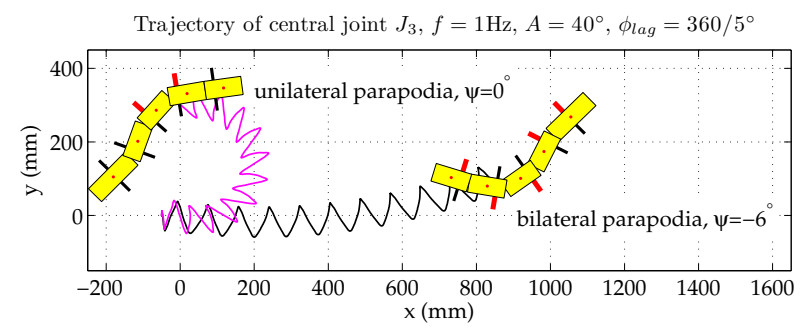

(b) turning gait simulations: polychaete pedundulatory mode

Fig. 5. Simulation results: (a) Forward gait simulations of the mechanism implementing the two pedundulatory modes, as well as the purely undulatory mode. (b) Turning gait simulations of the polychaete pedundulatory mode. 
efficiently penetrating granular substrates. In order to investigate the effect of their size on the locomotor performance, two parapodia variants have been developed, whose effective area differs by about $760 \mathrm{~mm}^{2}$ (Fig. 6a). In addition, special instrumented units of the two parapodia variants have been developed (Fig. 6b), incorporating a set of four strain gauges (Showa N11-MA2-120-11) in a Wheatstone-bridge setup, appropriately configured to measure the normal component $F_{N}^{p_{j}}$ of the force generated by the parapodium [1].

When the robot crawls over sand on the body segments' flat underside, a tail-to-head body wave is required for forward undulatory propulsion [1]. In order to alter the characteristics of its frictional interaction with the locomotion environment, and hence the propulsive mode and/or performance, different modules may be attached underneath the body segments. For example, when longitudinally-mounted wheeled modules are used, for movement over relatively smooth surfaces, forward propulsion is obtained by headto-tail body waves [4]. The effect of blade-bearing modules, similar to skates, is also under investigation [1]-[3].

\section{EXPERIMENTAL RESULTS}

\section{A. Locomotion on Sand}

Results from experiments of locomotion over sand with the Nereisbot implementing the two proposed pedundulatory modes, as well as purely undulatory gaits, are presented here and compared with simulation-derived results. This is a set of experimental data distinct from, but in agreement to, those obtained with the first version of the prototype, for the polychaete mode alone, in [1].

Experimental setup: The robot was placed inside a box (measuring $1.7 \mathrm{~m} \times 1.7 \mathrm{~m}$ ), which was uniformly filled with about $35 \mathrm{~mm}$ of fine seashore sand (mean particle diameter: $0.6 \mathrm{~mm}$ ). The experiments were conducted with the mechanism crawling on the body segments' flat underside, over a range of values for $A\left(20^{\circ}-60^{\circ}\right)$, with $f=1 \mathrm{~Hz}$, $\phi_{\text {lag }}=2 \pi / 5 \mathrm{rad}$, and utilizing the two parapodia variants of Fig. 6a. The robot was powered by an external $7.0 \mathrm{~V}$ power supply, while colored markers, placed on top of the tail $\left(J_{1}\right)$ and central $\left(J_{3}\right)$ joints of the mechanism, allowed the reconstruction of the respective joints' trajectories, via post-processing of the experiment videos.

Finally, corresponding simulations of the system were carried out in SIMUUN, replicating the experimental parameters, and incorporating the frictional data below.
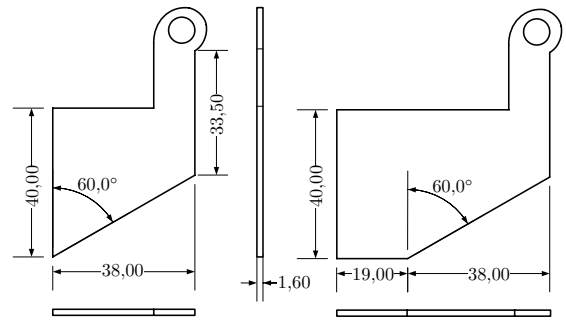

(a)

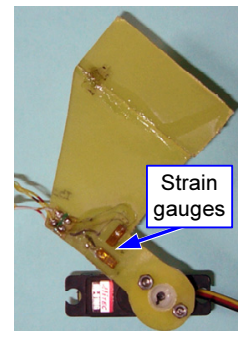

(b)
Fig. 6. (a) Dimensions (in $\mathrm{mm}$ ) of the two parapodia variants. (b) The larger variant of the instrumented parapodium, shown mounted on its motor.

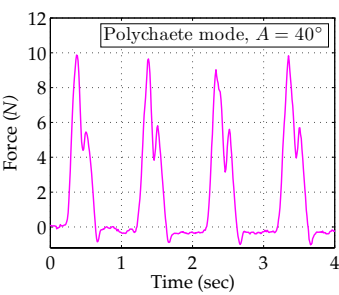

(a)

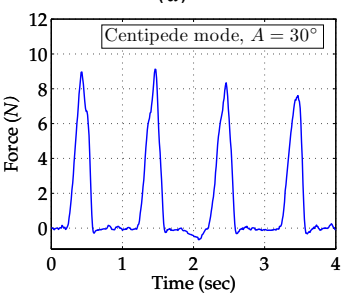

(b)

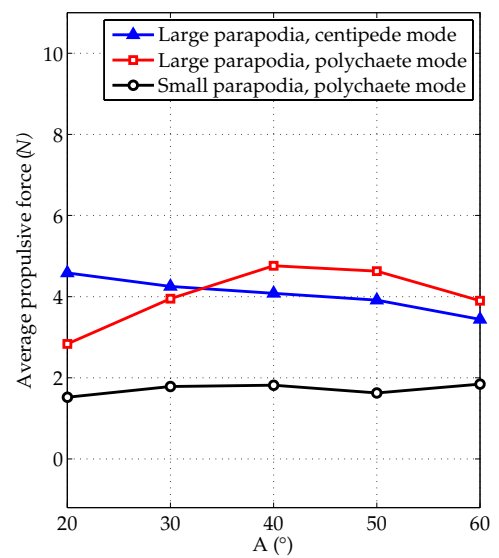

(c)
Fig. 7. Force data, obtained with the larger variant of the instrumented parapodium mounted on the tail segment of the mechanism, for forward pedundulatory locomotion on sand: Indicative data sets for (a) polychaeteand (b) centipede-mode. Positive force values correspond to forward thrust exertion. (c) Average generated propulsive forces (results from the smaller parapodium variant are also included for comparison).

Frictional force measurements: Prior frictional measurements [1] have indicated that a simple Coulomb friction model can be employed for the interaction of the body segments with sand, where for the flat-underside configuration of the mechanism, $\mu_{T}=0.518$ and $\mu_{N}=0.463$. Therefore, for our experimental setup, the frictional resistance in the tangential direction is slightly higher than in the normal direction; hence, body undulations will preferentially generate movement in the direction of wave propagation.

Data regarding the forces generated by the parapodia are provided by a series of pedundulatory forward gait experiments with the instrumented parapodia units mounted on the tail segment of the mechanism (the relevant data for the smaller parapodia are provided in [1], while results for the larger variant are summarized in Fig. 7). The obtained data indicate that, for both pedundulatory modes, the specified parapodial motions are properly coordinated with the corresponding body wave for thrust generation when in contact with the substrate (see Fig. 7a-b). Furthermore, the increased propulsive forces of the larger parapodia, compared to the smaller ones, are also confirmed (Fig. 7c). For each of the two parapodia variants, the results were subsequently used to specify the normal force coefficient $f_{N}$ (here presumed common among all of the mechanism's parapodia, since force data were only available from a single segment) of the Coulomb-like model employed for the frictional interaction of the parapodia with sand (cf. Section III-B). Due to the shape and movement of the parapodia, the magnitude of the tangentially-oriented frictional forces (specified through the coefficient $f_{T}$ ) may be considered negligible.

Undulatory mode: Experiments involving no parapodial activity confirmed that, as suggested by the SIMUUN simulations, forward propulsion for the flat-underside configuration of the prototype is by a tail-to-head body wave (1). The stride length attained in the purely undulatory forward gait was 


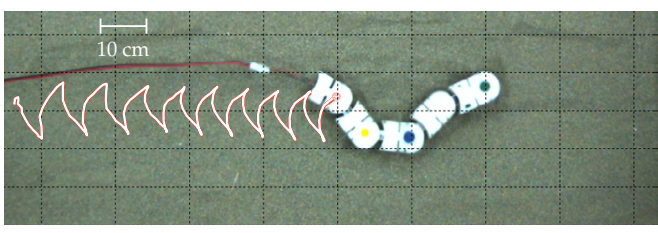

undulatory mode, $A=60^{\circ}$

Fig. 8. Forward undulatory gait: Nereisbot experimental results. The trace shows the trajectory of the tail joint $J_{1}$.

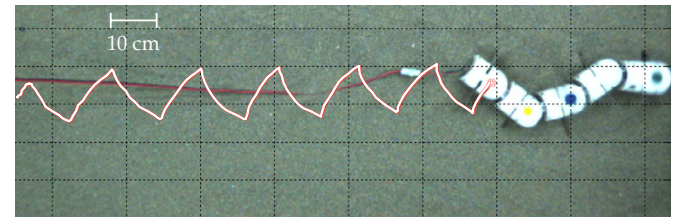

(a) polychaete pedundulatory mode, small parapodia, $A=60^{\circ}$

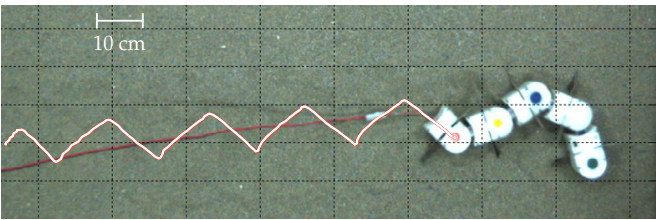

(b) polychaete pedundulatory mode, large parapodia, $A=60^{\circ}$

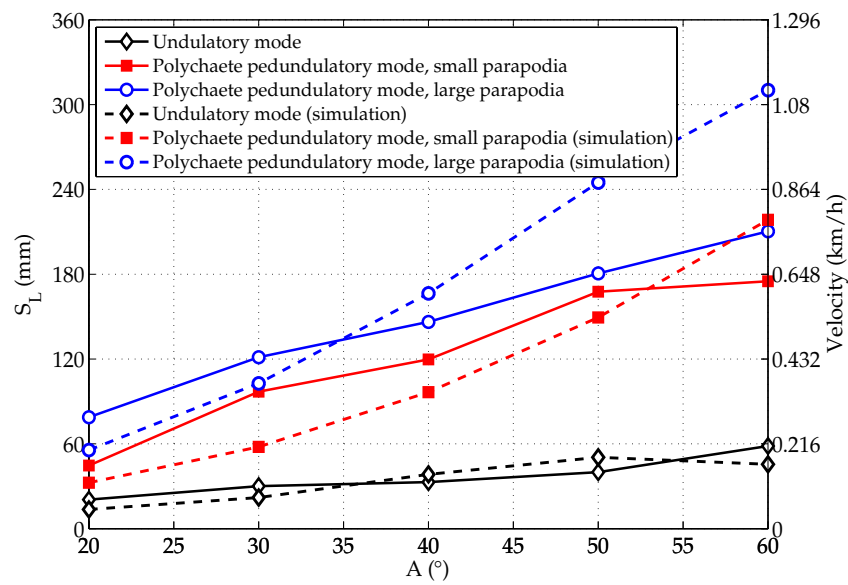

(c) summary of stride length results

Fig. 9. Forward polychaete-like pedundulatory gait on sand: (a)-(b) Nereisbot experimental results, for the two parapodia variants, indicating the trajectory of the tail joint $J_{1}$. (c) Experimental (solid lines) vs. simulationderived (dashed lines) stride length estimates of the robot. The results of the purely undulatory mode are also provided, for comparison purposes.

found to increase with the amplitude of joint oscillations, for the range of $A$ values tested, reaching a maximum of $S_{L} \simeq 65 \mathrm{~mm}$ when $A=60^{\circ}$ (see Figs. 8 and $9 \mathrm{c}$ ).

Polychaete pedundulatory mode: The polychaete-like pedundulatory gaits were obtained by employing (1)-(2) for motion control of the prototype, where guidelines for setting the parameters $g_{j}^{p}$ and $r_{j}$ are provided by the simulations described in Section IV-B. For the forward gait, indicative robot trajectories, obtained with the two variants of the parapodia, are shown in Fig. 9a-b, while, in Fig. 9c, the attained stride lengths are compared with those obtained by the purely undulatory mode, as well as with the corresponding stride lengths provided by the SIMUUN simulations.

The different methods for obtaining turning motions with the pedundulatory system, identified in Section IV, have also been experimentally validated. Indicative trajectories, shown in Fig. 10, illustrate the effect of the angular offset $\psi$ on the robot's turning direction and turning radius.

Centipede pedundulatory mode: The centipede-like pedundulatory gaits were obtained by employing (3)-(4) for motion control of Nereisbot. Indicative forward gait trajectories, obtained with the two variants of the parapodia, are shown in Fig. 11a-b. Estimates of the robot's attained stride lengths are summarized in Fig. $11 \mathrm{c}$, along with the corresponding stride lengths provided by the simulations. Indicative results from the turning gait experiments are shown in Fig. 12.

Discussion: The experimental results demonstrate both the validity of the proposed locomotion principles and the significantly increased performance associated with the pedundulatory motion control strategies. The attained stride length $S_{L}$, and thus the average velocity $v=f S_{L}$ of the robot, are significantly increased, particularly when employing the larger parapodia, compared to those attained in the purely undulatory mode. The robot reaches velocities up to $0.76 \mathrm{~km} / \mathrm{h}$ for the polychaete mode and $0.64 \mathrm{~km} / \mathrm{h}$ for the centipede mode. For equivalent experimental conditions, consistently higher stride lengths are obtained with the polychaete mode, compared to the centipede mode; this is also suggested by the simulation results.

Compared to the original Nereisbot prototype, the additional pair of parapodia (installed on the head segment) was found to noticeably improve the locomotion performance on sand, increasing the maximum stride length, attained with the smaller parapodia in polychaete mode, from $S_{L} \simeq 130 \mathrm{~mm}$ (as reported in [1]) to around $175 \mathrm{~mm}$ for the present version. Moreover, the increased propulsive forces generated by the larger parapodia variant (see Fig. 7c), are also demonstrated to yield further performance improvements on sand, throughout the range of experimental conditions investigated here, attaining a maximum stride length of about $210 \mathrm{~mm}$ for the polychaete mode and $175 \mathrm{~mm}$ for the centipede mode.

The experimental results also demonstrate, to a large extent, the validity of our computational models, which are successful both in capturing the main qualitative characteristics of the investigated locomotion strategies, and in providing adequate quantitative predictions for the robot performance. This is also manifested in the similarities between the shape of the simulation trajectories in Fig. 5a

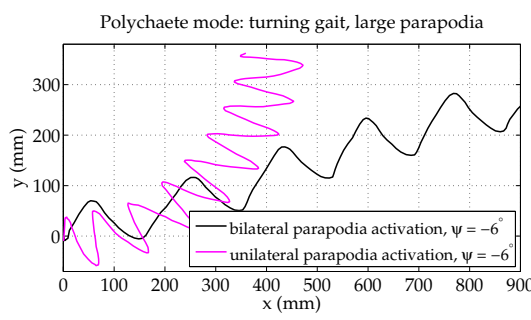

(a) unilateral vs. bilateral parapodia activation

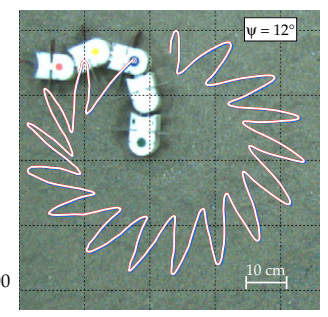

(b) unilateral parapodia activation
Fig. 10. Turning polychaete-like pedundulatory gait on sand: Nereisbot experimental results, obtained with the large parapodia, for $A=50^{\circ}$. The traces show the trajectory of the central joint $J_{3}$. 


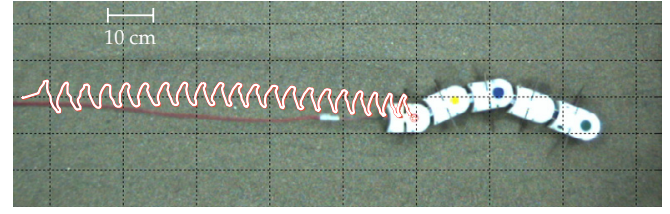

(a) centipede pedundulatory mode, small parapodia, $A=30^{\circ}$

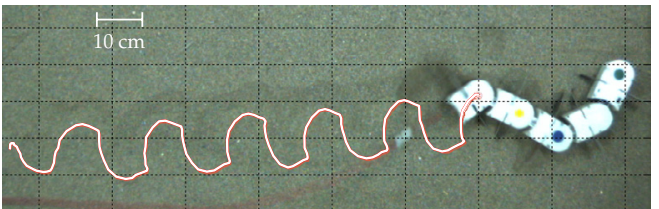

(b) centipede pedundulatory mode, large parapodia, $A=60^{\circ}$

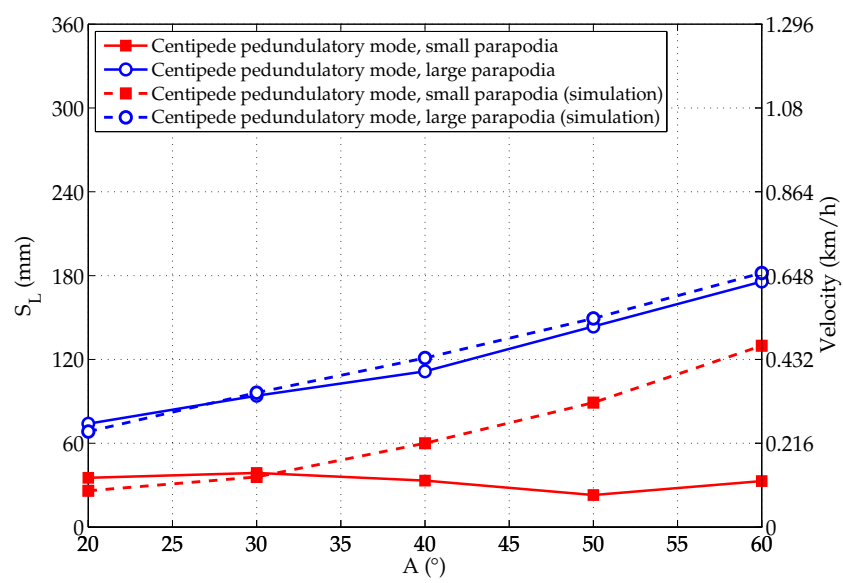

(c) summary of stride length results

Fig. 11. Forward centipede-like pedundulatory gait on sand: (a)-(b) Nereisbot experimental results, for the two parapodia variants, indicating the trajectory of the tail joint $J_{1}$. (c) Experimental (solid lines) vs. simulationderived (dashed lines) stride length estimates of the robot.

and the shape of the experimental trajectories in Figs. 8, 9 and 11. Any inconsistencies with the experimental data could be attributed, at least in part, to the simplified nature of the employed friction models, which cannot describe the whole range of complex phenomena and interactions, tribological or otherwise (e.g., sand shifting or sand compaction), occurring between the robot and the highly unstructured granular substrate considered in the present experimental study. Obtaining force data with the instrumented parapodium mounted on all of the robot's segments, in order to generate a more detailed profile of the thrust produced by the parapodia along the body of the mechanism, is expected to further improve the accuracy of the simulations' quantitative predictions.

The main discrepancy to the above points is to be found in the experimental results for the centipede pedundulatory mode with the smaller parapodia, where, as the joint oscillation amplitude is increased above $30^{\circ}$, the locomotion performance becomes significantly degraded, even in comparison with the purely undulatory mode (Fig. 11c). This may be related to the fact that, for the flat-underside configuration of the prototype, body undulations tend to move the robot in the direction of wave propagation; hence, the head-to-tail body wave employed in the centipede mode will, in fact, tend to counter-act the propulsive forces of the parapodia.

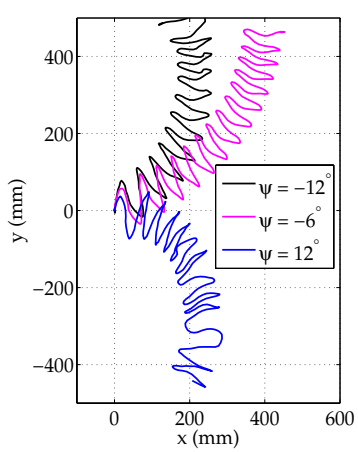

(a) small parapodia

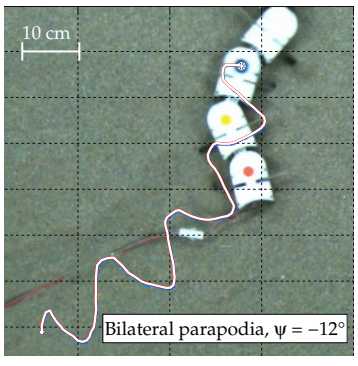

(b) large parapodia
Fig. 12. Turning centipede-like pedundulatory gait on sand: Nereisbot experimental results, obtained for bilateral parapodia activation, with $A=$ $50 \mathrm{deg}$. The traces show the trajectory of the central joint $J_{3}$.

The effect of this is more pronounced when the smaller parapodia are used and as the amplitude of body waves is increased. Under these conditions, based on our observations, a significant amount of sand shifting and/or compaction is generated, degrading the efficacy of locomotion, as well as the applicability of our computational models.

\section{B. Locomotion on Other Unstructured Substrates}

Additional experiments, described here, have been carried out with Nereisbot, in order to assess the performance of pedundulatory locomotion over (i) grass lawn (average grass height: $\sim 6 \mathrm{~cm}$ ), and (ii) ploughed soil (Fig. 13a-b). Since these substrates are, to a large extent, non-yielding, the motion range of the parapodia was reduced, so that ground contact would be made by their inner lower edge alone, thus avoiding rocking upwards motions of the robot, as well as excessive actuator stress. In these outdoors experiments, the prototype was powered by an on-board $7.4 \mathrm{~V}$ battery pack, and the average velocity attained with the two proposed pedundulatory modes, in forward locomotion (where $f=$ $1 \mathrm{~Hz}$ ), was estimated as a function of $A$. The obtained results, summarized in Fig. 13c, demonstrate the effectiveness of Nereisbot in traversing these substrates. For lawn grass, in particular, which presents relatively low frictional resistance to the crawling body, velocities as high as $0.9 \mathrm{~km} / \mathrm{h}$ and 0.74 $\mathrm{km} / \mathrm{h}$ were attained for the polychaete- and the centipedelike modes, respectively. Performance over the challenging environment of ploughed soil was also noticeable, reaching a maximum of about $0.65 \mathrm{~km} / \mathrm{h}$ in polychaete mode. Much like for sand, velocity was found to increase with $A$, while, between the two pedundulatory motion control schemes, higher velocities were attainable by the polychaete mode.

\section{CONCLUSIONS}

The combination of undulatory-based and appendagebased locomotion, as exploited by polychaete annelids and centipedes, has inspired the development of the novel class of pedundulatory robots, and associated motion control schemes thereof, which are presented here. The increased performance and significant potential of the proposed pedundulatory modes, particularly when compared to purely undulatory locomotion, has been studied through simulations 


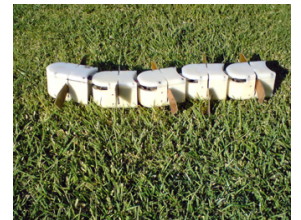

(a)

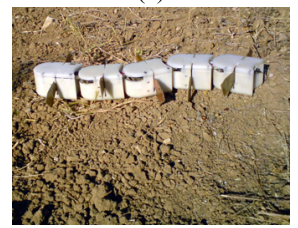

(b)

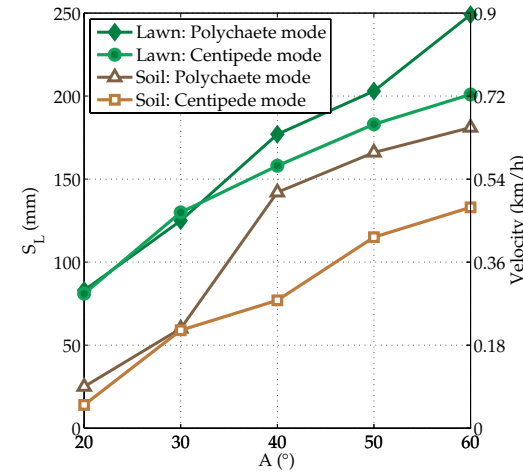

(c)
Fig. 13. Experiments of Nereisbot locomotion on (a) grass lawn, and (b) ploughed soil. (c) Attained velocities and equivalent stride lengths for the two pedundulatory modes, as a function of the joint oscillation amplitude.

and demonstrated experimentally, over sand, grass lawn and ploughed soil. The computational models developed predict adequately both qualitative and quantitative aspects of all these different modes of locomotion.

The reported findings suggest a performance advantage of the polychaete mode over the centipede mode, at least in terms of attainable velocities in the environments tested. Investigations considering additional performance indices (e.g., power consumption, path-clearing abilities, compatibility with sensor-based guidance schemes), are expected to provide a more thorough comparative assessment of the two modes. In this context, it is however noteworthy that our robotic platform can switch between the three presented modes (two pedundulatory and one undulatory) merely by altering the motion control strategy (i.e., without physically modifying the prototype), so as to, e.g., select on-the-fly the most appropriate one for different stages of an undertaken "mission".

Furthermore, additional (preliminary) experiments with Nereisbot indicate that pedundulatory locomotion is also effective (i) over a variety of other terrains, including granular substrates (e.g., pebbles, gravel), (ii) over various types of elastic and deformable materials (e.g., elastic/foam/fabric sheets), and (iii) in environments or situations where pathclearing is required for advancement (e.g., through tall vegetation, or with the robot partly submerged in sand). Optimizing the design, actuation mechanism and motion profile of the parapodia, either for best matching a specific locomotion environment, or for all-round performance of the robot, is therefore of interest. Similar investigations are underway regarding the modulation of the frictional interface between the body segments and the locomotion substrate, in order to, e.g., generate eel/snake-like modes of purely undulatory locomotion by retrograde body waves.

Closed-loop schemes, based on sensory feedback from the parapodia could also be explored, for, e.g., fine-tuning online the timing of the parapodia activation, so as to optimize thrust generation under all conditions. Finally, the generation of reactive behaviors [4], [5] for pedundulatory systems will also be considered.

\section{ACKNOWLEDGMENTS}

The authors thank N. Pateromichelakis and K. Karakasiliotis for their technical assistance. Related papers and videos can be found at the Web site www.ics.forth.gr/tsakiris

\section{REFERENCES}

[1] M. Sfakiotakis, D. P. Tsakiris, and K. Karakasiliotis, "Polychaete-like pedundulatory robotic locomotion," in Proc. IEEE Int. Conf. Robot. Autom., Roma, Italy, 2007, pp. 269-274.

[2] G. La Spina, M. Sfakiotakis, D. P. Tsakiris, A. Menciassi, and P. Dario, "Polychaete-like undulatory robotic locomotion in unstructured substrates," IEEE Trans. Robot., vol. 6, pp. 1200-1212, 2007.

[3] D. P. Tsakiris, M. Sfakiotakis, A. Menciassi, G. La Spina, and P. Dario, "Polychaete-like undulatory robotic locomotion," in Proc. IEEE Int. Conf. Robot. Autom., Barcelona, Spain, 2005, pp. 3029-3034.

[4] M. Sfakiotakis and D. P. Tsakiris, "A biomimetic centering behavior for undulatory robots," Int. J. Robot. Res., vol. 26, no. 11-12, pp. 1267-1282, 2007.

[5] — - "Neuromuscular control of reactive behaviors for undulatory robots," Neurocomputing, vol. 70, no. 10-12, pp. 1907-1913, 2007.

[6] A. A. Transeth, R. I. Leine, C. Glocker, and K. Y. Pettersen, "3-d snake robot motion: Nonsmooth modeling, simulations, and experiments," IEEE Trans. Robot., vol. 24, no. 2, pp. 361-376, 2008.

[7] K. Lipkin, I. Brown, A. Peck, H. Choset, J. Rembisz, P. Gianfortoni, and A. Naaktgeboren, "Differentiable and piecewise differentiable gaits for snake robots," in Proc. IEEE/RSJ Int. Conf. Intell. Robots Syst., San Diego, USA, 2007, pp. 1864-1869.

[8] M. Nilsson, "Serpentine locomotion on surfaces with uniform friction," in Proc. IEEE/RSJ Int. Conf. Intell. Robots Syst., Sendai, Japan, 2004, pp. 1751-1755.

[9] S. Hirose and E. Fukushima, "Snakes and strings: New robotic components for rescue operations," Int. J. Robot. Res., vol. 23, no. 4/5, pp. 341-349, 2004.

[10] M. Saito, M. Fukaya, and T. Iwasaki, "Modeling, analysis, and synthesis of serpentine locomotion with a multilink robotic snake," IEEE Control Syst. Mag., vol. 22, no. 1, pp. 64-81, 2002.

[11] A. Crespi, A. Badertscher, A. Guignard, and A. J. Ijspeert, "AmphiBot I: an amphibious snake-like robot," Robot. Auton. Syst., vol. 50, no. 4, pp. 163-175, 2005.

[12] J. Cortes, S. Martinez, J. P. Ostrowski, and K. A. McIsaac, "Optimal gaits for dynamic robotic locomotion," Int. J. Robot. Res., vol. 20, no. 9 , pp. 707-728, 2001.

[13] A. J. Ijspeert, A. Crespi, and J. Cabelguen, "Simulation and robotics studies of salamander locomotion. Applying neurobiological principles to the control of locomotion in robots," Neuroinf., vol. 3, no. 3, pp. 171-196, 2005.

[14] J. Gray, "Annelids," in Animal Locomotion. London: Weidenfeld \& Nicolson, 1968, pp. 377-410.

[15] R. Brusca and G. Brusca, Invertebrates. Sunderland: Sinauer Associates, 1990.

[16] R. B. Clark and D. J. Tritton, "Swimming mechanisms in nereidiform polychaetes," J. Zool., vol. 161, pp. 257-271, 1970.

[17] B. Anderson, J. Shultz, and B. Jayne, "Axial kinematics and muscle activity during terrestrial locomotion of the centipede, Scolopendra heros," J. Exp. Biol., vol. 198, no. 5, pp. 1185-1195, 1995.

[18] R. Full, "Invertebrate locomotor systems," in The Handbook of Comparative Physiology, W. Dantzler, Ed. Oxford University Press, 1997, pp. 853-930.

[19] J. G. E. Lewis, The Biology of Centipedes. Cambridge: Cambridge University Press, 1981.

[20] M. Sfakiotakis and D. P. Tsakiris, "SIMUUN: A simulation environment for undulatory locomotion," Int. J. Model. Simul., vol. 26, no. 4, pp. 4430-4464, 2006.

[21] S. Hirose, Biologically Inspired Robots: Snake-Like Locomotors and Manipulators. New York: Oxford University Press, 1993.

[22] J. P. Ostrowski and J. Burdick, "The geometric mechanics of undulatory robotic locomotion," Int. J. Robot. Res., vol. 17, no. 7, pp. 683-701, 1998.

[23] S. Ma, "Development of a creeping locomotion snake-robot," Int. J. Robot. Automat., vol. 17, no. 4, pp. 146-153, 2002. 